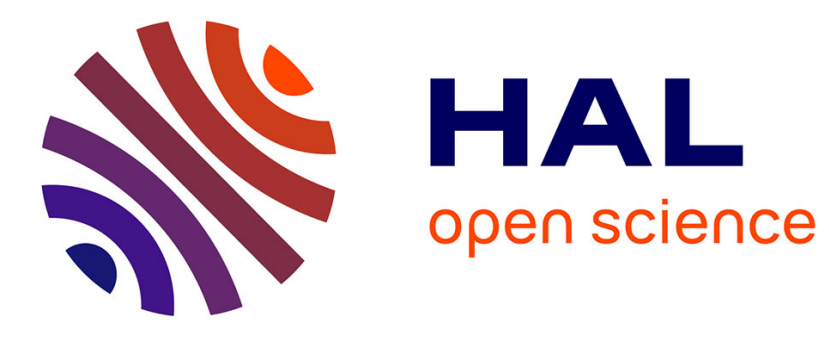

\title{
Model predictive control suitable for closed-loop re-identification
}

\author{
Alejandro Hernan Gonzalez, Antonio Ferramosca, Germán A. A Bustos, \\ Jacinto Luis L Marchetti, Mirko Fiacchini, Darci Odloak
}

\section{To cite this version:}

Alejandro Hernan Gonzalez, Antonio Ferramosca, Germán A. A Bustos, Jacinto Luis L Marchetti, Mirko Fiacchini, et al.. Model predictive control suitable for closed-loop re-identification. Systems and Control Letters, 2014, 69, pp.23-33. 10.1016/j.sysconle.2014.03.007 . hal-01010865

\section{HAL Id: hal-01010865 https://hal.science/hal-01010865}

Submitted on 5 Jul 2021

HAL is a multi-disciplinary open access archive for the deposit and dissemination of scientific research documents, whether they are published or not. The documents may come from teaching and research institutions in France or abroad, or from public or private research centers.
L'archive ouverte pluridisciplinaire HAL, est destinée au dépôt et à la diffusion de documents scientifiques de niveau recherche, publiés ou non, émanant des établissements d'enseignement et de recherche français ou étrangers, des laboratoires publics ou privés. 


\title{
Model predictive control suitable for closed-loop re-identification
}

\author{
A.H. González ${ }^{\mathrm{a}}$, A. Ferramosca ${ }^{\mathrm{a}}$, G.A. Bustos ${ }^{\mathrm{a}}$, J.L. Marchettia ${ }^{\mathrm{a}}$, M. \\ Fiacchini $^{\mathrm{b}}$, D. Odloak ${ }^{\mathrm{c}}$ \\ ${ }^{a}$ Institute of Technological Development for the Chemical Industry (INTEC), \\ CONICET-Universidad Nacional del Litoral (UNL). Güemes 3450, (3000) Santa Fe, \\ Argentina-alejgon@santafe-conicet.gov.ar,ferramosca@santafe-conicet.gov.ar, \\ gbustos@santafe-conicet.gov.ar \\ ${ }^{b}$ GIPSA-lab, Grenoble Campus, 11 rue des Mathématiques, BP 46, 38402 Saint Martin \\ d'Héres Cedex, France - mirko.fiacchini@gipsa-lab.fr \\ ${ }^{c}$ Department of Chemical Engineering, University of São Paulo, Av. Prof. Luciano \\ Gualberto, trv3380,61548 São Paulo,Brazil-odloak@usp.br
}

\begin{abstract}
The main problem of a closed-loop re-identification procedure is that, in general, the dynamic control and identification objectives are conflicting. In fact, to perform a suitable identification, a persistent excitation of the system is needed, while the control objective is to stabilize the system at a given equilibrium point. However, a generalization of the concept of stability, from punctual stability to (invariant) set stability, allows for a flexibility that can be used to avoid the conflict between these objectives. Taking into account that an invariant target set includes not only a stationary component, but also a transient one, the system could be excited without deteriorating the stability of the closed-loop. In this work, a MPC controller is proposed that assures the stability of invariant sets at the same time that a signal suitable for closed-loop re-identification is generated. Several simulation results show the propose controller formulation properties.
\end{abstract}

Keywords: Model predictive control, closed-loop identification, target set control, persistent excitation.

\section{Introduction}

Model predictive control (MPC) is typically implemented as a lower stage of a hierarchical control structure. The upper level stages are devoted to compute, by means of a stationary optimization, the targets that the dynamic control stage (MPC) should reach to economically optimize the operation of the process. Since both, the dynamic and stationary optimizations are model based optimizations, a periodic updating of the model parameters are desired to reach meaningful optimums. In this context, a re-identification procedure should be developed in a closed-loop fashion, since the process cannot be stopped each 
time an update is needed. As it is known, the main problem of a closed-loop identification is that the dynamic control objectives are incompatible with the identification objectives ([9]). In fact, to perform a suitable identification, a persistent excitation of the system modes is needed, while the controller takes this excitation as disturbance, and tries to reject this disturbance to stabilize the system.

From a general point of view, the closed-loop identification methods fall into the following main groups ([21]). The direct approach ignores the feedback law and identifies the open-loop system using measurements of the input and the output. The indirect approach identifies the closed-loop transfer function and determines the open-loop parameters subtracting the controller dynamic. To do that, the controller dynamics must be linear and known. The joint inputoutput approach takes the input and output jointly, as the output of a system produced by some extra input or set-point signal. Since the last two methods need the exact knowledge of a linear controller, they are not directly applicable for closed-loops under constrained MPC controllers.

Several strategies were developed to perform closed-loop re-identification under MPC controllers: [4] proposed a controller named Model Predictive Control and Identification (MPCI) where a persistent excitation condition is added by means of an additional constraint in the optimization problem. This strategy, which was explored later in [1], turns the MPC optimization problem nonconvex, and so, most of the well-known properties of the MPC formulation cannot be established. [22] proposed a strategy that manipulates the steady state target optimization (in the hierarchical MPC control structure) in order to excite the system. In the context of data driven MPC formulations (i.e., MPC that are designed to perform predictions directly from collected data), the subspace identification method is exclusively used ([18]). In [10], [23] and [17] several approaches are presented, where a closed-loop re-identification is needed to update the data for predictions. Though preliminary studies were made according to the trade-off between stability and excitation, no definitive results were presented.

In general, none of the reports cited in this section have shown results regarding the system stability of the MPC while the system is being re-identified. In this work, based on the concept of stability of an invariant set (as a generalization of stability of a point), a MPC controller with a extended domain of attraction is proposed, which assures stability at the same time that a persistent excitation can be generated to perform a closed-loop re-identification. Some preliminary results regarding the complete strategy presented in this work, were recently presented in [5].

Notation: Matrices $I_{n} \in \mathbb{R}^{n \times n}$ and $0_{n, m} \in \mathbb{R}^{n \times m}$ denote the identity matrix and the null matrix, respectively. A $C$-set is a convex and compact set that contains the origin. A proper $C$-set is a $\mathrm{C}$-set that contains the origin as an interior point. Consider two sets $\mathcal{U} \subseteq \mathbb{R}^{n}$ and $\mathcal{V} \subseteq \mathbb{R}^{n}$, containing the origin, and a real number $\lambda$. The Minkowski sum $\mathcal{U} \oplus \mathcal{V} \subseteq \mathbb{R}^{n}$ is defined by $\mathcal{U} \oplus \mathcal{V}=\{(u+v): u \in \mathcal{U}, v \in \mathcal{V}\}$; the set $(\mathcal{U} \backslash \mathcal{V}) \subseteq \mathbb{R}^{n}$ is defined as $\mathcal{U} \backslash \mathcal{V}=\{u: u \in \mathcal{U} \wedge u \notin \mathcal{V}\}$; and the set $\lambda \mathcal{U}=\{\lambda u: u \in \mathcal{U}\}$ is a scaled set of 
$\mathcal{U}$. $|v|_{\mathcal{V}}$ is the distance from $v$ to $\mathcal{V}$. The boundary of a set $\mathcal{U}$, is defined as $\partial \mathcal{U}$. Given a continuous function $\Psi: \mathbb{R}^{n} \rightarrow \mathbb{R}$, and $\gamma \geq 0$, the level set $\mathcal{N}[\Psi, \gamma]$ is defined by $\mathcal{N}[\Psi, \gamma]=\{x: \Psi(x) \leq \gamma\}$. $\mathbb{I}_{m: n}$ denotes the nonnegative integers from $m$ to $n$. Given $x \in \mathbb{R}^{n}$ and $y \in \mathbb{R}^{n},\|x-y\|_{M}^{2}=(x-y)^{T} M(x-y)$, with $M \in \mathbb{R}^{n \times n}$.

\section{Problem statement and preliminaries}

Consider a system described by a linear time-invariant discrete-time model

$$
x^{+}=A x+B u, \quad y=C x
$$

where $x \in \mathbb{R}^{n}$ is the system state, $x^{+}$is the successor state, $u \in \mathbb{R}^{m}$ is the current control, and $y \in \mathbb{R}^{p}$ is the system output. The state, the control input and the output at discrete-time instant $k$ are denoted as $x(k), u(k)$ and $y(k)$, respectively. The system is subject to hard constraints on state and input, $(x(k), u(k)) \in \mathcal{Z} \triangleq(\mathcal{X} \times \mathcal{U}) \subset \mathbb{R}^{n+m}$ for all $k \geq 0$, where $\mathcal{X} \subset \mathbb{R}^{n}$ and $\mathcal{U} \subset \mathbb{R}^{m}$. Furthermore, the following assumption holds:

Assumption 1. Matrix $A$ has all its eigenvalues strictly inside the unit circle, the pair $(A, B)$ is controllable and the state (corresponding to the true plant) is measured at each discrete-time instant. Furthermore, the set $\mathcal{X}$ is convex and closed, the set $\mathcal{U}$ is convex and compact and both contain the origin in their interior. For simplicity, $A \mathcal{X} \subseteq \lambda \mathcal{X}$, with $\lambda \in[0,1)$.

Previous to the controller formulation, some necessary definitions helpful to generalize the concepts of equilibrium and invariance are introduced. To simplify the notation, we denote system $x^{+}=A x+B u,(x, u) \in \mathcal{Z}$ as Nonautonomous system $\left(\mathcal{N}_{\text {sys }}\right)$ and system $x^{+}=A x+B \kappa(x),(x, \kappa(x)) \in \mathcal{Z}$, where $\kappa(x)$ is a state feedback, as Controlled system $\left(\mathcal{C}_{\text {sys }}\right)$. Accordingly, for a given sequence of control inputs, $\mathbf{u}=\{u(0), \cdots, u(j-1)\}$ and a given initial state $x(0)=x$, the solution of $\mathcal{N}_{\text {sys }}$ will be denoted as: $x(j)=\phi(j ; x, \mathbf{u})=A^{j} x(0)+\sum_{i=0}^{j-1} A^{j-i-1} B u(i), j \in \mathbb{I}_{\geq 1}$. Similarly, for a given initial state $x(0)=x$, the solution of $\mathcal{C}_{\text {sys }}$ will be denoted as: $x(j)=$ $\phi_{\kappa}(j ; x)=A^{j} x(0)+\sum_{i=0}^{j-1} A^{j-i-1} B \kappa x(i), j \in \mathbb{I}_{\geq 1}$.

Definition 1. (Control Equilibrium Set - CES) A set $\Omega \subseteq \mathcal{X}$ is a control equilibrium set for $\mathcal{N}_{\text {sys }}$, if for every point $x \in \Omega$ the condition $x^{+}=x$ holds for some $u \in \mathcal{U}$.

The maximal CES, $\mathcal{X}_{s s}$, is given by $\mathcal{X}_{s s}=(G B \mathcal{U}) \cap \mathcal{X}$, where $G=\left(I_{n}-A\right)^{-1}$. In case of controlled systems, $\mathcal{C}_{\text {sys }}$, we simply say that a Control Equilibrium set $\Omega$ is an Equilibrium Set - (ES), with $u=\kappa(x)$. The proper generalization of the concept of equilibrium point is not the concept of equilibrium set, as a mere aggregation of steady-state points, but the concept of invariant set (associated to an equilibrium set), in the sense that both, the equilibrium point and the invariant set are geometric entities such that, if the system reaches them, it remains in them indefinitely ([2], [11], [13]): 
Definition 2. ( $\lambda$-Control Invariant Set - $\lambda$-CIS) A proper $C$-set $\Omega \subseteq \mathcal{X}$ is $\lambda$-control invariant, with $\lambda \in[0,1]$, for $\mathcal{N}_{\text {sys }}$, if $x \in \Omega$ implies $x^{+} \in \lambda \Omega$, for some $u \in \mathcal{U}$.

Again, in case of controlled systems, $\mathcal{C}_{\text {sys }}$, a $\lambda$-Control Invariant set is simply a $\lambda$-Invariant Set - ( $\lambda$-IS $)$, with $u=\kappa(x)$. Furthermore, if $\lambda=1$, the sets are simply Invariant sets, and if $\lambda \in[0,1)$, the sets are known as Contractive sets. The concept of invariant set, as a generalization of an equilibrium point, makes possible the generalization of the concept of attractivity of an equilibrium point. Then, we can define the attractivity of an IS set as follows ([20]):

Definition 3. (Local attractivity of an IS set) The IS set $\Omega \subset \mathcal{X}$ is locally attractive for $\mathcal{C}_{\text {sys }}$ if for each $x$ in a vicinity of $\Omega$ (that we call the domain of attraction $)$, it follows that $\left|\phi_{\kappa}(j ; x)\right|_{\Omega} \rightarrow 0, \phi_{\kappa}(j ; x) \in \mathcal{X}, \kappa\left(\phi_{\kappa}(j ; x)\right) \in \mathcal{U}$ as $j \rightarrow \infty$.

\section{Target invariant set for identification}

The objective of this section is to propose a set (in the state space) that is invariant under the excitation procedure necessary to perform a suitable identification and, at the same time, can be used as attractive target set (generalized equilibrium) by an MPC controller. As known, to estimate a model from measured input and output data, each (controllable) mode of the system must be excited. To do that, the excitation input signal must contain enough variability. This property is generally indicated by the notion of persistence of excitation ([15]). The persistent excitation input sequences might be of several forms, going from a Pseudo-Random Binary Signal (PRBS) signal to a Filtered Pseudo Gaussian White Noise Signal. A recent formulation proposed a filtered Gaussian inputs signal specifically designed for MPC ([14]). Independently of the form, the persistent excitation sequences have two main properties: they are bounded, belonging to a compact set smaller than $\mathcal{U}$, and more subtle, they have a persistent-variability behavior. Regarding the first property, we define:

Definition 4. (Excitation input set, EIS). An input proper $C$-set $\mathcal{U}^{t} \subset \mathcal{U} \subset$ $\mathbb{R}^{m}$, with enough size to excite the system will be denoted as excitation input set.

The set $\mathcal{U}^{t}$ defines a class of sequences $\mathbf{u}=\left\{u(0), \cdots, u\left(T_{i d}-1\right)\right\}$ - denoted by $\mathcal{C}_{\mathcal{U}^{t}}$ - such that $u(i) \in \mathcal{U}^{t}$ for $i \in \mathbb{I}_{0: T_{i d}-1}$, where $T_{i d}$ is the length of the data necessary to perform a suitable identification. Now, regarding the second property of persistent excitation, we define the class of all feasible sequences that fulfill the persistent-variability condition (see [8], [4], for the explicit expression of this condition). This class - that we call the persistent excitation class, $\mathcal{C}_{p e}$ - is made by sequences of the form $\mathbf{u}_{p e}=\left\{u_{p e}(0), \cdots, u_{p e}\left(T_{i d}-1\right)\right\}, u_{p e}(i) \in \mathcal{U}^{t}$, $i \in \mathbb{I}_{0: T_{i d}-1}$. Notice that, following the latter classification, $\mathcal{C}_{p e}$ is in $\mathcal{C}_{\mathcal{U}^{t}}$, but there is an entire class of input sequences that are in $\mathcal{C}_{\mathcal{U}^{t}}$ and not in $\mathcal{C}_{p e}$. For instance, a constant sequence of $T_{i d}$ elements, $\overline{\mathbf{u}}=\{\bar{u}, \cdots, \bar{u}\}$, with $\bar{u} \in \mathcal{U}^{t}$, belongs to $\mathcal{C}_{\mathcal{U}^{t}}$ but does not belong to $\mathcal{C}_{p e}$. 
The solution of $\mathcal{N}_{\text {sys }}$ for a particular sequence $\mathbf{u}_{p e}$ in $\mathcal{C}_{p e}$, and an initial state $x(0)=x$, will be denoted as $x(j)=\phi\left(j ; x, \mathbf{u}_{p e}\right), j \in \mathbb{I}_{\geq 1}$ (persistently excited system). Notice that once a particular input sequence is selected, then the system evolves autonomously, in the sense that no manipulated controls modify the system evolution. We can now define a set in the state space that is invariant to the effect of any persistent excitation sequence.

Definition 5. ( $\lambda$-Invariant set for Persistent Excitation) A proper $C$-set $\mathcal{X}^{p e} \subseteq \mathcal{X}$ is a $\lambda$-invariant set for persistent excitation, with $\lambda \in[0,1]$, for $\mathcal{N}_{\text {sys }}$, if $x \in \mathcal{X}^{p e}$ implies $\phi\left(j ; x, \mathbf{u}_{p e}\right) \in \lambda \mathcal{X}^{p e}$, for $j \in \mathbb{I}_{1: T_{i d}}$, for all $\mathbf{u}_{p e} \in \mathcal{C}_{p e}$.

The set $\mathcal{X}^{p e}$ is well defined and it is what we need to formulate the MPC for re-identification. However, $\mathcal{X}^{p e}$ is not easy to characterize, given that the Persistent Excitation condition is not related to a spatial condition, but to a dynamic requirement. Next, an approximation of $\mathcal{X}^{p e}$ that is useful for MPC requirements is proposed.

\subsection{A MPC target set that is an approximation of $\mathcal{X}^{p e}$}

Instead of associate an invariant set, $\mathcal{X}^{p e}$, to the class $\mathcal{C}_{p e}$, we could relax the definition and associate an invariant set to the class $\mathcal{C}_{\mathcal{U}^{t}}$, which contains $\mathcal{C}_{p e}$. So, the following definition arises:

Definition 6. ( $\lambda$-Invariant set for identification, $\lambda$-ISI) A proper $C$-set $\mathcal{X}^{t} \subseteq \mathcal{X}$ is a $\lambda$-invariant set for identification, with $\lambda \in[0,1]$, for $\mathcal{N}_{\text {sys }}$, if $x \in \mathcal{X}^{t}$ implies $\phi(j ; x, \mathbf{u}) \in \lambda \mathcal{X}^{t}$, for $j \in \mathbb{I}_{1: T_{i d}}$, for all $\mathbf{u} \in \mathcal{C}_{\mathcal{U}^{t}}$.

A $\lambda$-ISI set with $\lambda=1$ is simply an ISI set. Since by definition every input sequence of $\mathcal{C}_{p e}$ is in $\mathcal{C}_{\mathcal{U}^{t}}$, it follows that $\mathcal{X}^{p e} \subseteq \mathcal{X}^{t}$, which means that $\mathcal{X}^{t}$ is an outer "invariant" approximation of $\mathcal{X}^{p e}$. Clearly, an ISI set definition is a robust invariant set definition adapted to identification requirements. So, it is relatively easy to compute, and we can define the smallest ISI set, for a given system, which is given by the minimal ISI set ([13]): $\underline{\mathcal{X}}^{t}=\bigoplus_{i=0}^{\infty}\left(A^{i} B \mathcal{U}^{t}\right)$.

Remark 1. Although the set $\mathcal{X}^{t}$ could be easy to compute (for polytopic constraint sets), it could be much bigger than $\mathcal{X}^{p e}$. For instance, since Persistent Excitation signals cannot remain constant at a boundary value of $\mathcal{U}^{t}$, then the boundary regions of the set $\mathcal{X}^{t}$ (the points far from the origin) that corresponds to the equilibrium subspace $\mathcal{X}_{\text {ss }}$ are not in $\mathcal{X}^{p e}$. The methodical reduction of the size of set $\mathcal{X}^{t}$ (and so, of the conservatism of the formulation) to obtain accurate approaches of $\mathcal{X}^{\text {pe }}$ could be done in several ways, using for instance the concepts of probabilistic invariant sets ([12]) and other deterministic concepts as the Pontryagin difference, etc.

Next, some properties regarding the ISI sets are stated.

Property 1. A set $\mathcal{X}^{t}$ is a $\lambda$-ISI set for $\mathcal{N}_{\text {sys }}$ if and only if $A \mathcal{X}^{t} \oplus B \mathcal{U}^{t} \subseteq \lambda \mathcal{X}^{t}$. 
Property 2. Conisder a $\lambda$-ISI set $\mathcal{X}^{t}$, with $\lambda \in[0,1]$, for $\mathcal{N}_{\text {sys }}$, and a CES set, $\mathcal{X}_{s s}^{t}=G B \mathcal{U}^{t} \subseteq \mathcal{X}$, for the same system. Also consider a $\lambda$-IS set, $\Omega \subset \mathcal{X}$, with $\lambda \in[0,1)$, for the autonomous system $x^{+}=A x, x \in \mathcal{X}$. Then:

(1) $\mathcal{X}^{t}$ is also a $\lambda$-invariant set for persistent excitation, $\mathcal{X}^{\text {pe }}$ (contractivity of $\mathcal{X}^{t}$ under persistent excitation).

(2) For each $x$ in a vicinity of $\mathcal{X}^{t},\left|\phi\left(j ; x, \mathbf{u}_{p e}\right)\right|_{\mathcal{X}^{t}} \rightarrow 0, \phi\left(j ; x, \mathbf{u}_{p e}\right) \in \mathcal{X}$, as $j \rightarrow \infty$ (attractivity of $\mathcal{X}^{t}$ under persistent excitation).

(3) $\mathcal{X}_{s s}^{t} \subseteq \mathcal{X}^{t}$ and furthermore, if $m<n, \mathcal{X}_{s s}^{t} \subset \mathcal{X}^{t}$ ( $\mathcal{X}^{t}$ includes stationary and transient states).

(4) Provided that $\left(\mathcal{X}^{t} \oplus \Omega\right) \subset \mathcal{X}$, there exists a real $\delta \in(\lambda, 1)$, such that if $x \in\left(\mathcal{X}^{t} \oplus \Omega\right)$, then $\phi(j ; x, \mathbf{u}) \in \delta\left(\mathcal{X}^{t} \oplus \Omega\right)$ for all $j \in \mathbb{I}_{\geq 1}$, for all $\mathbf{u}_{p e} \in \mathcal{C}_{p e}$ (contractivity of $\mathcal{X}^{t} \oplus \Omega$ under persistent excitation).

(5) If $\delta \mathcal{X}^{t} \subset \mathcal{X}$, for a constant $\delta>1, \delta \mathcal{X}^{t}$ is also an $\lambda$-ISI set (contractivity of $\delta \mathcal{X}^{t}$ under persistent excitation).

The proofs of these properties are omitted for brevity.

\subsection{Target set $\mathcal{X}^{t}$ for model mismatch}

It should be noticed that the ISI set $\mathcal{X}^{t}$, which will be a parameter of the proposed MPC optimization cost, depends on the model. Since the excitation scenario is precisely given when we suspect that the current model is no longer accurate, a discussion about the effect of the model mismatch on the computation of $\mathcal{X}^{t}$ is needed. Two uncertainty descriptions - that are only particular descriptions - were selected to present robust ISI sets.

Parametric uncertainty

Consider a system given by

$$
x^{+}=A(w) x+B(w) u, \quad y=C(w) x, \quad w \in \mathcal{W} \subseteq \mathbb{R},
$$

where $A(w)$ and $B(w)$ are affine functions of $w$, i.e., $A(w)=A+w \bar{A}, B(w)=$ $B+w \bar{B}$ with $w$ belonging to the proper C-set $\mathcal{W} \subset \mathbb{R}$. Furthermore, assume that the Nominal model is given by $x^{+}=A\left(w_{N}=0\right) x+B\left(w_{N}=0\right) u=A x+B u$, and the unknown Real model, is given by $x^{+}=A\left(w_{R}\right) x+B\left(w_{R}\right) u$, for some $w_{R} \in \mathcal{W}$. In this context, there is a minimal ISI set $\underline{\mathcal{X}}^{t}(w)$ associated to each particular model. Now, consider the following Theorem:

Theorem 1. Consider a $\lambda$-ISI set $\mathcal{X}^{t}(w=0), \lambda \in[0,1)$, for $x^{+}=A x+B u$, $(x, u) \in \mathcal{Z}$. Then, there exists a non-empty set $\mathcal{W} \subset \mathbb{R}$ for which the set $\mathcal{X}^{t}(w=0)$ is an ISI set for $x^{+}=A(w) x+B(w) u,(x, u) \in \mathcal{Z}$, for all $w \in \mathcal{W}$.

Proof. Consider

$$
\begin{aligned}
A(w) \mathcal{X}^{t} \oplus B(w) \mathcal{U}^{t} & =(A+w \bar{A}) \mathcal{X}^{t} \oplus(B+w \bar{B}) \mathcal{U}^{t} \\
& \subseteq\left(A \mathcal{X}^{t} \oplus B \mathcal{U}^{t}\right) \oplus w\left(\bar{A} \mathcal{X}^{t} \oplus \bar{B} \mathcal{U}^{t}\right) \subseteq \lambda \mathcal{X}^{t} \oplus w \Theta
\end{aligned}
$$


where $\Theta \triangleq \bar{A} \mathcal{X}^{t} \oplus \bar{B} \mathcal{U}^{t}$. The first inclusion follows from Minkowski sum properties, while the second one follows from the fact that $\mathcal{X}^{t}(w=0)$ is a $\lambda$-ISI set, $\lambda \in[0,1)$, for the Nominal model. Now, since $\mathcal{X}^{t}$ and $\mathcal{U}^{t}$ are proper $\mathrm{C}$-sets, $\Theta$ is also a proper C-set. Then, a value $\bar{w}>0$ does exists such that $\bar{w} \Theta \subseteq(1-\lambda) \mathcal{X}^{t}$. Therefore, we have

$$
A(w) \mathcal{X}^{t} \oplus B(w) \mathcal{U}^{t} \subseteq \lambda \mathcal{X}^{t} \oplus \bar{w} \Theta \subseteq \lambda \mathcal{X}^{t} \oplus(1-\lambda) \mathcal{X}^{t}=\mathcal{X}^{t}
$$

Then, the non-empty set $\mathcal{W} \triangleq[0, \bar{w}]$ is such that $\mathcal{X}^{t}$ is an ISI set for $x^{+}=$ $A(w) x+B(w) u,(x, u) \in \mathcal{Z}$, for all $w \in \mathcal{W}$. Furthermore, assuming that $\Theta$ is 0 -simetric, a similar procedure can be followed for $-\bar{w}$. In this way, the (non-empty) set $\mathcal{W}$ is given now by $\mathcal{W} \triangleq[-\bar{w}, \bar{w}]$.

Next, based on the result in Theorem 1, a practical form to compute the robust ISI set is proposed.

Remark 2. (Practical computation of the robust ISI set) Property 2,(4) provides a way to obtain an ISI set like the one required by the hypotesis of Theorem 1. Consider a $\lambda$-IS set $\Omega_{\lambda}, \lambda \in[0,1)$, for the autonomous system $x^{+}=A(w=0) x=A x, x \in \mathcal{X}$, and take into account that $\xi \Omega_{\lambda}, \xi>0$, is also a (contractive) $\lambda$-IS set for the same system. Then, the set $\mathcal{X}^{t}=\mathcal{X}^{t}(\xi, \lambda) \triangleq$ $\underline{\mathcal{X}}^{t}(w=0) \oplus \xi \Omega_{\lambda}$, where $\underline{\mathcal{X}}^{t}(w=0)$ is the minimal ISI set for the Nominal system, is a $\delta$-ISI set, with $\delta \in(\lambda, 1)$, for the same Nominal system. Now, for given sets $\mathcal{W} \triangleq[-\bar{w}, \bar{w}]$ and $\mathcal{U}^{t}$, it is desirable to obtain the smallest robust ISI set $\mathcal{X}^{t}(\xi, \lambda)$, which can be done by solving the following problem:

$(\bar{\xi}, \bar{\lambda})=\min _{\xi, \lambda}\left\{(\xi, \lambda) \mid\left(A \mathcal{X}^{t}(\xi, \lambda) \oplus B \mathcal{U}^{t}\right) \oplus \bar{w}\left(\bar{A} \mathcal{X}^{t}(\xi, \lambda) \oplus \bar{B} \mathcal{U}^{t}\right) \subseteq \mathcal{X}^{t}(\xi, \lambda)\right\}$

The smallest robust ISI set is then given by $\mathcal{X}^{t}=\mathcal{X}^{t}(\bar{\xi}, \bar{\lambda}) \triangleq \underline{\mathcal{X}}^{t}(w=0) \oplus \bar{\xi} \Omega_{\bar{\lambda}}$.

Notice that the model described in (1) is not a mere scaling of matrices $A$ and $B$, since matrices $\bar{A}$ and $\bar{B}$ are in general different from the nominal matrices. So, notice that a general set $\tilde{X}$ containing all possible minimal invariant sets for the uncertainty system is not useful for a robust formulation of the MPC, since this set is not necessarily an ISI set for each model of the family.

\section{Additive disturbance uncertainty}

In this case we have a model of the form $x^{+}=A x+B u+D d$, where $D$ is the disturbance matrix, $d \in \mathcal{D}$ is the disturbance vector that describes the uncertainty and $\mathcal{D}$ is a proper $\mathrm{C}$-set. In such a case, by selecting a $\lambda$-ISI set $\mathcal{X}^{t}$, for the extended system

$$
x^{+}=A x+\left[\begin{array}{ll}
B & D
\end{array}\right]\left[\begin{array}{l}
u \\
d
\end{array}\right], \quad\left[\begin{array}{l}
u \\
d
\end{array}\right] \in \mathcal{U}^{t} \times \mathcal{D},
$$


we obtain a $\lambda$-ISI set for the uncertain system $x^{+}=A x+B u+D d,(x, u) \in$ $\mathcal{Z}$, for all $d \in \mathcal{D}$. This kind of disturbance can include output noise (i.e., identification noise), measurement noise, and other kinds of disturbances that cannot be described by the parametric uncertainty.

The two robust ISI sets presented in this subsection are ISI sets for uncertain systems. However, it should be noted that an explicit robust formulation of the proposed MPC strategy, which assures the feasible robust convergence, is not in the scope of the present approach, and is delayed for future works.

\section{MPC suitable for re-identification (main result)}

The objective of the proposed controller is to avoid the conflicts between excitation and control objectives: when the system is outside a target set, the target set is a control target; when the system is inside, the target set is a safe identification set, that is considered as a generalized equilibrium by the controller.

The key concept to achieve such an MPC controller is to propose an MPC cost that (1) penalizes the distance to the target set, and (2) is null at every point of the target set, in the same way a standard MPC cost is null at the desired punctual target. This means that the controller does not make a difference between any two points of the target, or, in other words, the controller leaves the system in open loop when it enters the target set. We will start this class of MPC controllers with a controller for tracking control equilibrium sets (CES), which is known as zone control MPC controllers.

\section{1. $M P C$ for tracking control equilibrium sets (CES)}

Consider the EIS set $\mathcal{U}^{t} \subset \mathcal{U}$, and the associated CES set $\mathcal{X}_{s s}^{t}=G B \mathcal{U}^{t} \subseteq \mathcal{X}$. This controller is formulated following the strategy proposed in $[3,6]$, usually known as zone control. The cost function is given by

$V_{N}^{C E S}\left(x, \mathcal{X}_{s s}^{t} ; \mathbf{u}, u_{s s}, x_{s s}\right)=\sum_{j=0}^{N-1}\left(\left\|x(j)-x_{s s}\right\|_{Q}^{2}+\left\|u(j)-u_{s s}\right\|_{R}^{2}\right)+\gamma\left|x_{s s}\right|_{\mathcal{X}_{s s}^{t}}$,

where $Q>0$ and $R \geq 0$ are penalization matrices, $\gamma>0$ is a real number, $|x|_{\mathcal{X}^{t}}$ is the distance function (from $x$ to $\mathcal{X}_{s s}^{t}$ ) and $N$ is the control horizon. Furthermore, $x_{s s}=G B u_{s s}$ is a free stationary state in $\mathcal{X}_{s s}=G B \mathcal{U}$. For any current state $x \in \mathcal{X}$, the optimization problem $P_{N}^{C E S}\left(x, \mathcal{X}_{s s}^{t}\right)$ to be solved is given by: 
Problem $P_{N}^{C E S}\left(x, \mathcal{X}_{s s}^{t}\right)$

$$
\begin{aligned}
& \min _{\mathbf{u}, u_{s s}, x_{s s}} V_{N}^{C E S}\left(x, \mathcal{X}_{s s}^{t} ; \mathbf{u}, u_{s s}, x_{s s}\right) \\
& x(0)=x, \\
& x(j+1)=A x(j)+B u(j), \quad j \in \mathbb{I}_{0: N-1} \\
& x(j) \in \mathcal{X}, u(j) \in \mathcal{U}, \quad j \in \mathbb{I}_{0: N-1} \\
& u(j)=u_{s s}, \quad j \in \mathbb{I}_{N-1: \infty} \\
& x_{s s}=G B u_{s s} \text {. }
\end{aligned}
$$

In this optimization problem, $x$ and $\mathcal{X}_{s s}^{t}$ are the parameters, while the sequence $\mathbf{u}=\{u(0), \cdots, u(N-1)\}$ and $x_{s s}, u_{s s}$, are the optimization variables. Notice that $x_{s s}, u_{s s}$ are only forced to represent an equilibrium point, but are free to assume any feasible equilibrium value. The control law, derived from the application of a receding horizon policy, is given by $\kappa_{N}\left(x, \mathcal{X}_{s s}^{t}\right)=u^{0}(0 ; x)$, where $u^{0}(0 ; x)$ is the first element of the solution sequence $\mathbf{u}^{0}(x)$. Now the following Theorem can be established:

Theorem 2. Consider that Assumption 1 holds, and consider a given CES $\mathcal{X}_{s s}^{t}=G B \mathcal{U}^{t} \subseteq \mathcal{X}$, with an associated input set $\mathcal{U}^{t}$. Then, $\mathcal{X}_{s s}^{t}$ is an $E S$ for the closed-loop system $x(j)=\phi_{\kappa_{N}}\left(j ; x, \mathcal{X}_{s s}^{t}\right), x(0)=x, j \in \mathbb{I}_{\geq 1}$. Furthermore, $\mathcal{X}_{s s}^{t}$ is locally attractive for the same closed-loop system, with a domain of attraction given by $\mathcal{X}$.

Proof. The proof can be seen in $[3,6]$.

Notice that this formulation comes directly from the output tracking problem, if we consider output zones of the form $C \mathcal{X}_{s s}^{t}$ (being a particular case the set-point tracking, if set $\mathcal{X}_{s s}^{t}$ is a single point). The idea to steer the system to an equilibrium set (with independence of the single value of the equilibrium point), suggests the possibility to extend this concept to invariant sets, which also include transient states.

\section{2. $M P C$ for tracking invariant sets for identification (ISI)}

Now, a generalization of the MPC controller for tracking CES sets is presented. The idea is to track and reach sets - i.e., ISI sets - that not only include stationary states, but also transient states, which is necessary to perform a proper identification. We start with a quite general formulation, that is particularized in the next subsections to different applicable cases. The nominal case will be considered, although the extension to the use of robust ISI sets is direct. Consider an ISI set $\mathcal{X}^{t}$ for $\mathcal{N}_{\text {sys }}$, and the associated EIS set $\mathcal{U}^{t}$. Also consider the following definition

Definition 7. (Generalized distance stage cost function) A generalized distance function $d_{\mathcal{X}^{t}}(x)$, from $x$ to the ISI set $\mathcal{X}^{t}$, is a function with the following properties: $(1) d_{\mathcal{X}^{t}}(x)$ is convex and continuous for all $x \in \mathcal{X},(2)$ $d_{\mathcal{X}^{t}}(x)=0$ for all $x \in \mathcal{X}^{t}$, (3) $d_{\mathcal{X}^{t}}(x)>0$ for all $x \in \mathcal{X} \backslash \mathcal{X}^{t},(4) d_{\mathcal{X}^{t}}(x)$ 
is a Lyapunov function for the excited system $x(j)=\phi\left(j ; x, \mathbf{u}_{p e}\right), x(0)=x$, $j \in \mathbb{I}_{\geq 1}$, for all $x \in \mathcal{X} \backslash \mathcal{X}^{t}$.

The proposed controller cost function will be given by:

$$
V_{N}^{I S I}\left(x, \mathcal{X}^{t} ; \mathbf{u}\right)=\sum_{j=0}^{N-1}\left(\alpha d_{\mathcal{X}^{t}}(x(j))+\beta d_{\mathcal{U}^{t}}(u(j))\right)+d_{\mathcal{X}^{t}}^{t e r}(x(N)) .
$$

where $\alpha$ and $\beta$ are positive real numbers and $d_{\mathcal{X}^{t}}^{\text {ter }}(\cdot)$ is a terminal cost function defined on $\mathcal{X}^{\text {ter }}$, and $\mathcal{X}^{\text {ter }} \subseteq \mathcal{X}^{t}$ is the terminal set where the terminal state $x(N)$ is forced to belong. As usual in MPC design, we need to select a local control action (defined by some conditions) that will act for predictions inside the terminal set. At this moment we simply define a fixed control action $\hat{u}=$ $\hat{u}(x) \in \mathcal{U}^{t}$. Then, associated to this local control action, the terminal set and terminal cost must fulfill the following conditions:

$$
\begin{aligned}
\mathcal{X}^{t e r} \text { is IS for } x^{+} & =A x+B \hat{u}, x \in \mathcal{X} \\
d_{\mathcal{X}^{t}}^{t e r}(A x+B \hat{u})-d_{\mathcal{X}^{t}}^{t e r}(x) & \leq-\alpha d_{\mathcal{X}^{t}}(x), \forall x \in \mathcal{X}^{\text {ter }} \\
d_{\mathcal{X}^{t}}^{\text {ter }}(x) & =0, \forall x \in \mathcal{X}^{t} .
\end{aligned}
$$

Different forms for the local control action, the terminal cost and terminal set will be presented in section 5.3. For any current state in the set of states that can be feasibly steered to $\mathcal{X}^{\text {ter }}$ in $N$ steps (the $N$-step controllable set to $\mathcal{X}^{\text {ter }}$ ), $x \in \mathcal{X}_{N}\left(\mathcal{X}^{t e r}\right)$, the optimization problem $P_{N}^{I S I}\left(x, \mathcal{X}^{t}\right)$ to be solved is given by:

Problem $P_{N}^{I S I}\left(x, \mathcal{X}^{t}\right)$

$$
\begin{array}{clr}
\min _{\mathbf{u}} & V_{N}^{I S I}\left(x, \mathcal{X}^{t} ; \mathbf{u}\right) & \\
\text { s.t. } & & \\
& x(0)=x, & \\
& x(j+1)=A x(j)+B u(j), & \\
& x(j) \in \mathcal{X}, u(j) \in \mathcal{U}, & \\
& x(N) \in \mathbb{X}_{0: N-1} \\
& (N) . &
\end{array}
$$

The main differences between this problem and problem $P_{N}^{C E S}\left(x, \mathcal{X}_{s s}^{t}\right)$ are: (1) the target set is now an ISI set, which includes transient states (i.e., the states can be moved in a certain region with null control cost and without control actions), and (2) the generalized distance functions are now used to penalize the state and input trajectory in the MPC cost. The controller derived from this formulation assures the convergence of the closed-loop system to the ISI set $\mathcal{X}^{t}$, and once the system is there, it only assures that it will remain indefinitely in it. The next Theorems formalize these properties:

Theorem 3. Let Assumption 1 hold, and consider an ISI set $\mathcal{X}^{t} \subseteq \mathcal{X}$, with an associated EIS set $\mathcal{U}^{t}$. Then, $\mathcal{X}^{t}$ is an IS set for the closed-loop system $x(j)=\phi_{\kappa_{N}}\left(j ; x, \mathcal{X}^{t}\right), x(0)=x, j \in \mathbb{I}_{\geq 1}$. 
Proof. Consider a state $x \in \mathcal{X}^{t}$. Then, by definition of ISI sets, any input sequence $\hat{\mathbf{u}}=\{u(0), \ldots, u(N-1)\}$, with $u(j) \in \mathcal{U}^{t}$, for $j \in \mathbb{I}_{0: N-1}$, produces a sequence of states that remain in $\mathcal{X}^{t}$. So, considering the definition of the generalized distance function, the input sequence is a trivial optimal solution of $P_{N}^{I S I}\left(x, \mathcal{X}^{t}\right)$, with $V_{N}^{I S I}\left(x, \mathcal{X}^{t} ; \hat{\mathbf{u}}\right)=0$. On the other hand, any input sequence $\hat{\mathbf{u}}$ with $u(j) \notin \mathcal{U}^{t}$, for some $j \in \mathbb{I}_{0: N-1}$, produces a cost $V_{N}^{I S I}\left(x, \mathcal{X}^{t} ; \hat{\mathbf{u}}\right) \geq 0$. This means that necessarily $u^{0}(0 ; x) \in \mathcal{U}^{t}$. This proves that the MPC cost $V_{N}^{I S I}\left(x, \mathcal{X}^{t} ; \mathbf{u}\right)$ is null along every trajectory starting in an initial state inside $\mathcal{X}^{t}$, and furthermore, $u^{0}(0 ; x)$ is a control input inside $\mathcal{U}^{t}$. From this fact, it directly follows that $\mathcal{X}^{t}$ is an IS set for the MPC closed-loop system.

Theorem 4. Let Assumption 1 hold, and consider an ISI set $\mathcal{X}^{t} \subseteq \mathcal{X}$, with an associated EIS set $\mathcal{U}^{t}$. Then, $\mathcal{X}^{t}$ is locally attractive for the closed-loop system $x(j)=\phi_{\kappa_{N}}\left(j ; x, \mathcal{X}^{t}\right), x(0)=x, j \in \mathbb{I}_{\geq 1}$, with a domain of attraction given by $\mathcal{X}_{N}\left(\mathcal{X}^{\text {ter }}\right)$.

Proof. Consider a state $x \in \mathcal{X}_{N}\left(\mathcal{X}^{\text {ter }}\right) \backslash \mathcal{X}^{t}$, at a given time $k$. Consider also the solution defined for this state, $\mathbf{u}^{0}(x)=\left\{u^{0}(0 ; x), \cdots, u^{0}(N-1 ; x)\right\}$, and the corresponding state sequence $\mathbf{x}^{0}(x)=\left\{x^{0}(0 ; x), \cdots, x^{0}(N ; x)\right\}$, with $x(N ; x)^{0} \in$ $\mathcal{X}^{\text {ter }}$. The cost function of Problem $P_{N}^{I S I}\left(x, \mathcal{X}^{t}\right)$ corresponding to $\mathbf{u}^{0}(x)$ is given by

$$
\begin{aligned}
V_{N}^{I S I^{0}}\left(x, \mathcal{X}^{t}\right) & =V_{N}^{I S I}\left(x, \mathcal{X}^{t} ; \mathbf{u}^{0}(x)\right) \\
& =\sum_{j=0}^{N-1}\left(\alpha d_{\mathcal{X}^{t}}\left(x^{0}(j ; x)\right)+\beta d_{\mathcal{U}^{t}}\left(u^{0}(j ; x)\right)\right)+d_{\mathcal{X}^{t}}^{t e r}\left(x^{0}(N ; x)\right) .
\end{aligned}
$$

Now, consider the successor state $x^{+}=A x+B u^{0}(0 ; x)$, at time $k+1$, which is obtained by applying the control law $\kappa_{N}\left(x, \mathcal{X}^{t}\right)=u^{0}(0 ; x)$, and define the following sequence: $\hat{\mathbf{u}}=\left\{u^{0}(1 ; x), \cdots, u^{0}(N-1 ; x), \hat{u}\right\}$, where $\hat{u}$ is the local control action. Since no model mismatch is considered for predictions, the successor states $x^{+}$is equal to $x^{0}(1 ; x)$. This solution has an associated state sequence $\hat{\mathbf{x}}=\left\{x^{0}(1 ; x), \cdots, x^{0}(N ; x), \hat{x}\right\}$, where $\hat{x}=A x^{0}(N ; x)+B \hat{u}$. Since $x^{0}(N ; x) \in \mathcal{X}^{\text {ter }}$ and $\mathcal{X}^{\text {ter }}$ is an IS set for the system $x^{+}=A x+B \hat{u}, x \in \mathcal{X}$, then $\hat{x} \in \mathcal{X}^{\text {ter }}$. Therefore, sequence $\hat{\mathbf{u}}$ is a feasible solution for problem $P_{N}^{I S I}\left(x, \mathcal{X}^{t}\right)$ at time $k+1$. The cost function of Problem $P_{N}^{I S I}\left(x^{+}, \mathcal{X}^{t}\right)$, at $k+1$, for the sequence $\hat{\mathbf{u}}$, is given by

$V_{N}^{I S I}\left(x^{+}, \mathcal{X}^{t} ; \hat{\mathbf{u}}\right)=\sum_{j=0}^{N-1}\left(\alpha d_{\mathcal{X}^{t}}\left(x\left(j ; x^{+}\right)\right)+\beta d_{\mathcal{U}^{t}}\left(u\left(j ; x^{+}\right)\right)\right)+d_{\mathcal{X}^{t}}^{t e r}\left(x\left(N ; x^{+}\right)\right)$,

where $x^{+}=x^{0}(1 ; x)$. So, this cost can be written as a function of $x$,

$$
\begin{aligned}
V_{N}^{I S I}\left(x^{+}, \mathcal{X}^{t} ; \hat{\mathbf{u}}\right)= & \sum_{j=1}^{N-1}\left(\alpha d_{\mathcal{X}^{t}}\left(x^{0}(j ; x)\right)+\beta d_{\mathcal{U}^{t}}\left(u^{0}(j ; x)\right)\right) \\
& +\left(\alpha d_{\mathcal{X}^{t}}\left(x^{0}(N ; x)\right)+\beta d_{\mathcal{U}^{t}}(\hat{u})\right)+d_{\mathcal{X}^{t}}^{t e r}(\hat{x})
\end{aligned}
$$


If we compare now the proposed feasible cost at time $k+1$ with the optimal one at time $k$, we have:

$$
\begin{aligned}
V_{N}^{I S I}\left(x^{+}, \mathcal{X}^{t} ; \hat{\mathbf{u}}\right)- & V_{N}^{I S I^{0}}\left(x, \mathcal{X}^{t}\right)=-\alpha d_{\mathcal{X}^{t}}\left(x^{0}(0 ; x)\right)-\beta d_{\mathcal{U}^{t}}\left(u^{0}(0 ; x)\right) \\
& +\left(\alpha d_{\mathcal{X}^{t}}\left(x^{0}(N ; x)\right)+\beta d_{\mathcal{U}^{t}}(\hat{u})\right)+d_{\mathcal{X}^{t}}^{t e r}(\hat{x})-d_{\mathcal{X}^{t}}^{t e r}\left(x^{0}(N ; x)\right)
\end{aligned}
$$

Since $\hat{u} \in \mathcal{U}^{t}$ by definition, $d_{\mathcal{U}^{t}}(\hat{u})=0$. Furthermore, since $x(N ; x)^{0} \in \mathcal{X}^{\text {ter }}$ and $\hat{x} \in \mathcal{X}^{\text {ter }}$, then - by conditions $(5), d_{\mathcal{X}^{t}}^{\text {ter }}(\hat{x})-d_{\mathcal{X}^{t}}^{\text {ter }}\left(x(N ; x)^{0}\right) \leq-\alpha d_{\mathcal{X}^{t}}\left(x(N ; x)^{0}\right)$. Therefore,

$$
\begin{aligned}
V_{N}^{I S I}\left(x^{+}, \mathcal{X}^{t} ; \hat{\mathbf{u}}\right)-V_{N}^{I S I^{0}}\left(x, \mathcal{X}^{t}\right)= & -\alpha d_{\mathcal{X}^{t}}\left(x^{0}(0 ; x)\right)-\beta d_{\mathcal{U}^{t}}\left(u^{0}(0 ; x)\right) \\
& +\alpha d_{\mathcal{X}^{t}}\left(x^{0}(N ; x)\right)-\alpha d_{\mathcal{X}^{t}}\left(x^{0}(N ; x)\right) \\
= & -\alpha d_{\mathcal{X}^{t}}\left(x^{0}(0 ; x)\right)-\beta d_{\mathcal{U}^{t}}\left(u^{0}(0 ; x)\right) .
\end{aligned}
$$

Now, by optimality of the solution to Problem $P_{N}^{I S I}\left(x^{+}, \mathcal{X}^{t}\right)$, at $k+1$, we have $V_{N}^{I S I^{0}}\left(x^{+}, \mathcal{X}^{t}\right) \leq V_{N}^{I S I}\left(x^{+}, \mathcal{X}^{t} ; \hat{\mathbf{u}}\right)$, and so

$$
V_{N}^{I S I^{0}}\left(x^{+}, \mathcal{X}^{t}\right)-V_{N}^{I S I^{0}}\left(x, \mathcal{X}^{t}\right) \leq-\alpha d_{\mathcal{X}^{t}}\left(x^{0}(0 ; x)\right)-\beta d_{\mathcal{U}^{t}}\left(u^{0}(0 ; x)\right) .
$$

Since the generalized distance is a positive definite function, (7) implies that $x^{0}(0 ; x)$ tends to $\mathcal{X}^{t}$ and $u^{0}(0 ; x)$ tends to $\mathcal{U}^{t}$ as $k \rightarrow \infty$, and so, the system converges to the desired ISI set.

Theorems 3 and 4 suggest that an extra requirement to the input, such as a persistent excitation requirement, could be included in the proposed cost function. In fact, what is shown is that, when the system is in $\mathcal{X}^{t}$, any bounded external input disturbance that does not bring the input outside $\mathcal{U}^{t}$, will not produce any effect on the controller.

\subsection{Including the exciting mode}

Given the properties of the latter formulation, several strategies could be followed to excite the system under control, when the state is in $\mathcal{X}^{t}$, and furthermore, to update the model parameters by means of an on line estimation. To do the first, we could add an exciting constraint, as the one used in [16], [4], or even a penalization term into the optimization cost, as the one proposed in [9]. To update the model parameters, a simple recursive least-square (RLS) algorithm ([15]) is recommended for practical applications.

However, since the focus of the present work is mainly in the separation of the objectives in the MPC formulation, we will simply consider a precomputed sequence $\mathbf{u}_{p e}$ in $\mathcal{C}_{p e}$ (which is also in $\mathcal{C}_{\mathcal{U}^{t}}$ ) as desired input trajectory. On the other side, no parameter update methods will be discussed. The proposed persistent excitation $M P C$ cost function is as follows:

$$
V_{N}^{E X C}\left(x, \mathcal{X}^{t}, \mathbf{u}_{p e}, k ; \mathbf{u}\right)=(1-\rho(x)) V_{N}^{I S I}\left(x, \mathcal{X}^{t} ; \mathbf{u}\right)+\rho(x)\left\|u(0)-u_{p e}(k)\right\|,
$$

where $\rho(x)=1$ if $x \in \mathcal{X}^{t}$, and $\rho(x)=0$, otherwise. The proposed strategy is a switching control strategy, in which the switching is operated by function 
$\rho(x)$. Notice that the state $x$ corresponding to the true plant is assumed to be known, and so, the switching function operates on true state measurements. For any initial state $x$ in $\mathcal{X}_{N}\left(\mathcal{X}^{t e r}\right)$, at a given time step $k$, the optimization problem $P_{N}^{E X C}\left(x, \mathcal{X}^{t}, \mathbf{u}_{p e}, k\right)$, to be solved at each time instant $k$, is given by:

$$
\begin{aligned}
& \text { Problem } P_{N}^{E X C}\left(x, \mathcal{X}^{t}, \mathbf{u}_{p e}, k\right) \\
& \min _{\mathbf{u}} V_{N}^{E X C}\left(x, \mathcal{X}^{t}, \mathbf{u}_{p e}, k ; \mathbf{u}\right) \\
& \text { s.t. } \\
& x(0)=x, \\
& x(j+1)=A x(j)+B u(j), \quad j \in \mathbb{I}_{0: N-1} \\
& x(j) \in \mathcal{X}, u(j) \in \mathcal{U}, \quad j \in \mathbb{I}_{0: N-1} \\
& x(N) \in \mathcal{X}^{\text {ter }}
\end{aligned}
$$

Notice that the function $\rho(x)$ is a discontinuous function necessary to cancel the persistent excitation in case that an external disturbance takes the system away from the invariant set $\mathcal{X}^{t}$. The following Theorem formalizes the properties of the proposed MPC controller:

Theorem 5. Let Assumption 1 hold, and consider an ISI set $\mathcal{X}^{t}$ and a persistent excitation sequence $\mathbf{u}_{p e}$ in $\mathcal{C}_{p e}$. Then, for any initial state $x \in \mathcal{X}^{t}$, the system controlled by the receding horizon $M P C$ control law $\kappa_{N}\left(x, \mathcal{X}^{t}\right)=u^{0}(0 ; x)$, will be persistently excited inside $\mathcal{X}^{t}$, i.e., $x(j)=\phi_{\kappa_{N}}\left(j ; x, \mathcal{X}^{t}, \mathbf{u}_{p e}, j\right)=\phi\left(j ; x, \mathbf{u}_{p e}\right)$, $x(0)=x, j \in \mathbb{I}_{\geq 1}$. Furthermore, for any initial state $x \in \mathcal{X}_{N}\left(\mathcal{X}^{\text {ter }}\right) \backslash \mathcal{X}^{t}$, the closed-loop converges to $\mathcal{X}^{t}$.

Proof. i) Let us consider an initial state $x \in \mathcal{X}_{N}\left(\mathcal{X}^{t}\right) \backslash \mathcal{X}^{t}$. Then, $\rho(x)=0$, and so Problem $P_{N}^{E X C}\left(x, \mathcal{X}^{t}, \mathbf{u}_{p e}, k\right)$ is equivalent to Problem $P_{N}^{I S I}\left(x, \mathcal{X}^{t}\right)$. Furthermore, for Theorem 4 , the closed-loop system will admissibly converge to $\mathcal{X}^{t}$.

ii) Consider now an initial state $x \in \mathcal{X}^{t}$. Then $\rho(x)=1$, and the persistent excitation penalization is activated. Furthermore, $(1-\rho(x)) V_{N}^{I S I}\left(x, \mathcal{X}^{t} ; \mathbf{u}\right)=0$. This means that the cost of problem $P_{N}^{E X C}\left(x, \mathcal{X}^{t}, \mathbf{u}_{p e}, k\right)$ will be:

$$
V_{N}^{E X C}\left(x, \mathcal{X}^{t}, \mathbf{u}_{p e}, k ; \mathbf{u}\right)=\left\|u(0)-u_{p e}(k)\right\| .
$$

Now, since $\mathcal{X}^{t}$ is an ISI set and the persistent excitation sequence $\mathbf{u}_{p e}$ is in $\mathcal{U}^{t}$, then, the system will remain inside $\mathcal{X}^{t}$. So, the cost can be admissibly canceled if and only if $u^{0}(0 ; x)=u_{p e}(k)$ for each time $k$. This guarantees the persistent excitation of the open-loop system.

Remark 3. Another (practical) alternative to implement the strategy is by defining a second $\lambda$-ISI set $\mathcal{X}^{t t}=\delta \mathcal{X}^{t}, \delta>1$. Then, if the state is in $\mathcal{X}^{t t} \backslash \mathcal{X}^{t}$, the system keeps in open-loop (the control action are forced to be null). The invariant condition of $\mathcal{X}^{t t}$ (property 2.(5)) assures that once the state enter $\mathcal{X}^{t t}$ it will keep in $\mathcal{X}^{t t}$, and furthermore, the attractivity condition of $\mathcal{X}^{t}$ (property 2.(2)) assures that the state will reach $\mathcal{X}^{t}$. 


\subsection{Operation of the loop}

Based on the discussion in 4.1, 4.2 and 4.3, the MPC controller operation will be presented. We have two Operation modes: (1) Control operation mode: in this mode no re-identification is needed, and the $M P C$ for tracking CES set is implemented (Problem $P_{N}^{C E S}\left(x, \mathcal{X}_{s s}^{t}\right)$ ); and (2) Re-identification operation mode: this mode is activated only when there is a suspect that the model is not working properly, and a re-identification is needed. In this mode the persistent excitation $M P C$ is used (Problem $\left.P_{N}^{E X C}\left(x, \mathcal{X}^{t}, \mathbf{u}_{p e}, k\right)\right)$.

Remark 4. Based on Theorem 5, the Re-identification operation mode has in addition two spatially separated modes. When the system is outside $\mathcal{X}^{t}$, it is positively steered to it by the controller; once it is inside $\mathcal{X}^{t}$, it cannot be steered outside this set by the controller, even in an uncertainty scenario as the one described in section 3.2. If an unknown moderate disturbance enters the system and takes the state outside $\mathcal{X}^{t}$ - and provided that the current state $x$ is measured or well estimated, then the controller will automatically switch, by means of function $\rho(x)$, to only control the system to $\mathcal{X}^{t}$. On the other hand, if a strong disturbance scenario arises, a Re-identification experiment has no sense, and it is recommended to pass to the Control operation mode.

\section{Candidates for generalized distance functions and the terminal costs}

Provided that the proposed MPC formulations are strongly based on the concept of generalized distance functions, two possible candidates that fulfills Definition 7 will be presented in this section.

\subsection{Distance from a point to a set}

Definition 8. Distance from a point to a set. Given an ISI set $\mathcal{X}^{t} \subset \mathcal{X}$, the distance from $x$ to $\mathcal{X}^{t}$ is defined as

$$
|x|_{\mathcal{X}^{t}} \triangleq \min _{\hat{x} \in \mathcal{X}^{t}}\|x-\hat{x}\|_{M}^{2}, \quad M>0 .
$$

Function $|x|_{\mathcal{X}^{t}}$ has the following properties:

Property 3. (1) $|x|_{\mathcal{X}^{t}}$ is convex and continuous for all $x \in \mathcal{X}$, (2) $|x|_{\mathcal{X}^{t}}=0$ for all $x \in \mathcal{X}^{t}$, (3) $|x|_{\mathcal{X}^{t}}>0$ for all $x \in \mathcal{X} \backslash \mathcal{X}^{t}$, (4) $\mathcal{N}\left[|x|_{\mathcal{X}^{t}}, \gamma\right]=\mathcal{X}^{t} \oplus \mathcal{B}_{\gamma}$, for all $x \in \mathcal{X} \backslash \mathcal{X}^{t}$, where $\mathcal{B}_{\gamma} \triangleq\left\{x \in \mathcal{X}:\|x\|_{M}^{2} \leq \gamma\right\}, \gamma>0$.

Property 4. Let Assumption 1 hold, and suppose that $\mathcal{X}^{t} \subseteq \mathcal{X}$ is an ISI set for $\mathcal{N}_{\text {sys. }}$. Furthermore, consider that matrix $M>0$, associated to the distance function, is such that $A^{T} M A-M=-Q$ for some $Q>0$. Then, the distance function $|x|_{\mathcal{X}^{t}}$ is a Lyapunov function for the excited system $x(j)=\phi\left(j ; x, \mathbf{u}_{p e}\right)$, $x(0)=x, j \in \mathbb{I}_{\geq 1}$, for all $x \in \mathcal{X} \backslash \mathcal{X}^{t}$. 
Proof. First, notice that the set $\mathcal{B}_{\gamma}$ defined in Property 3.(4), is a $\lambda$-IS for the system $x^{+}=A x, x \in \mathcal{X}$, with $\lambda \in[0,1)$, provided that matrix $M>0$ is such that $A^{T} M A-M=-Q$ for some $Q>0$. Therefore, from Property 2,(4), and Property 3.(4), it follows that the level sets $\mathcal{N}\left[|x|_{\mathcal{X}^{t}}, \gamma\right]=\mathcal{X}^{t} \oplus \mathcal{B}_{\gamma}$, with $\gamma>0$, are $\delta$-IS sets, $\delta \in(\lambda, 1)$, for the excited system $x(j)=\phi\left(j ; x, \mathbf{u}_{p e}\right), x(0)=x$, $j \in \mathbb{I}_{\geq 1}$, for all $x \in \mathcal{X} \backslash \mathcal{X}^{t}$ (we assume for simplicity that $\left(\mathcal{X}^{t} \oplus \mathcal{B}_{\gamma}\right) \subset \mathcal{X}$ ). Consider now a state $x \in \partial\left(\mathcal{X}^{t} \oplus \mathcal{B}_{\gamma}\right)$, for some $\gamma>0$, which implies that $|x|_{\mathcal{X}^{t}}=\gamma$. Then,

$$
x^{+}=(A x+B u) \in \delta\left(\mathcal{X}^{t} \oplus \mathcal{B}_{\gamma}\right), \quad \forall u \in \mathcal{U}^{t} .
$$

This implies that $\left|x^{+}\right|_{\mathcal{X}^{t}} \leq \delta \gamma$, an so, $\left|x^{+}\right|_{\mathcal{X}^{t}} \leq \delta|x|_{\mathcal{X}^{t}}$, with $\delta \in(\lambda, 1)$, which means that $|x|_{\mathcal{X}^{t}}$ is a Lyapunov function for the aforementioned system, for all $x \in \mathcal{X} \backslash \mathcal{X}^{t}$.

\subsection{Modified Minkowski functional}

The Minkowski functional ([2]) is defined as:

Definition 9. Given an ISI set $\mathcal{X}^{t} \subset \mathcal{X}$, the Minkowski functional $\Psi_{\mathcal{X}^{t}}$ associated to $\mathcal{X}^{t}$ is defined as

$$
\Psi_{\mathcal{X}^{t}}(x)=\inf \left\{\mu \geq 0: x \in \mu \mathcal{X}^{t}\right\} .
$$

The Minkowski functional has a number of useful properties ([2]). It also was already used as a part of MPC costs in works as [19] and [7]. However, the Minkowski functional is not null inside the set to which it is associated. To achieve this property, we need to introduce the modified Minkowski functional.

Definition 10. Modified Minkowski functional. Given a convex set $\mathcal{X}^{t} \subset$ $\mathcal{X}$ that includes the origin as an interior point, and a $\lambda$-IS, $\Omega$, with $\lambda \in[0,1)$, for the system $x^{+}=A x, x \in \mathcal{X}$, the modified Minkowski functional is defined as

$$
\hat{\Psi}_{\mathcal{X}^{t}}(x) \triangleq \inf \left\{\mu \geq 0: x \in \mathcal{X}^{t} \oplus \mu \Omega\right\}
$$

Function $\hat{\Psi}_{\mathcal{X}^{t}}(x)$ has the following properties:

Property 5. (1) $\hat{\Psi}_{\mathcal{X}^{t}}(x)$ is convex and continuous for all $x \in \mathcal{X},(2) \hat{\Psi}_{\mathcal{X}^{t}}(x)=$ 0 for all $x \in \mathcal{X}^{t},(3) \hat{\Psi}_{\mathcal{X}^{t}}(x)>0$ for all $x \in \mathcal{X} \backslash \mathcal{X}^{t},(4) \mathcal{N}\left[\hat{\Psi}_{\mathcal{X}^{t}}(x), \gamma\right]=\mathcal{X}^{t} \oplus \gamma \Omega$, for all $x \in \mathcal{X} \backslash \mathcal{X}^{t}, \gamma>0$.

Property 6. Let Assumption 1 hold, and suppose that $\mathcal{X}^{t} \subseteq \mathcal{X}$ is an ISI set for $\mathcal{N}_{\text {sys. }}$. Then, $\hat{\Psi}_{\mathcal{X}^{t}}(x)$ is a Lyapunov function for the excited system $x(j)=\phi\left(j ; x, \mathbf{u}_{p e}\right), x(0)=x, j \in \mathbb{I}_{\geq 1}$, for all $x \in \mathcal{X} \backslash \mathcal{X}^{t}$.

Proof. This proof follows a similar procedure to the one of Theorem 4, taking into account Property 5.(4), of the Modified Minkowski functional, and the $\lambda$-IS condition of $\Omega$.

Figure 1 shows a schematic plot of a Modified Minkowski functional and a Distance function - together to the level sets - associated to an arbitrary polytopic set. Notice that inside the set the functions are null. 


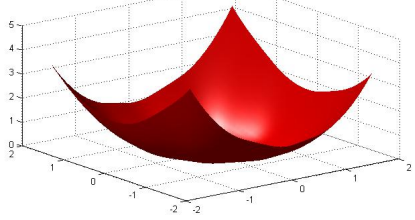

Distance Function

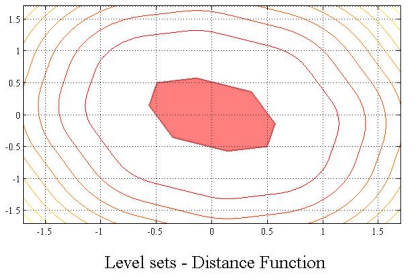

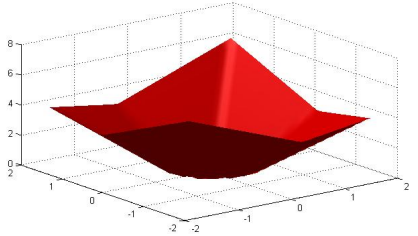

Modified Minkowski Function

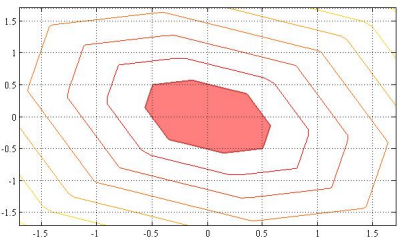

Level sets - Modified Minkowski Function

Figure 1: Descriptive plot of the Modified Minkowski Functional and the Distance Function associated to a given set.

\subsection{Several choices for the local control action and the terminal cost and set}

The easiest choice for the local control action, $\hat{u}$, is $\hat{u} \triangleq 0 \in \mathcal{U}^{t}$ (this choice is possible since $A$ is stable). Then, the terminal set and terminal cost are $\mathcal{X}^{\text {ter }} \triangleq \mathcal{X}$ and $d_{\mathcal{X}^{t}}^{\text {ter }}(x) \triangleq \delta d_{\mathcal{X}^{t}}(x(j)) \geq \sum_{j=0}^{\infty} \alpha d_{\mathcal{X}^{t}}(x(j)), x(0)=x$, respectively. Since $\mathcal{X}$ is an IS set for $x^{+}=A x$, by definition, then $\mathcal{X}^{\text {ter }}$ fulfills equality (4). On the other hand, since $d_{\mathcal{X}^{t}}(x)$ is a Lyapunov function for the system $x(j)=\phi\left(j ; x, \mathbf{u}_{p e}\right), x(0)=x, j \in \mathbb{I}_{1: N-2}$, for $x \in \mathcal{X} \backslash \mathcal{X}^{t}$ (Properties 4 and 6 ), and $u(j)=0 \in \mathcal{U}^{t}$, for $j \in \mathbb{I}_{N-1: \infty}$, it is possible to select a (large enough) real number $\delta>0$ such that $d_{\mathcal{X}^{t}}^{\text {ter }}(x)$ fulfills inequality (5) for all $x \in \mathcal{X}$. Equality (6) is fulfilled trivially. The drawback of this choice is that the proposed MPC solution could be far from optimality, since no control action is implemented for predictions beyond the horizon $N$.

The other choice for the local control action is to simply select any fixed $\hat{u} \in \mathcal{U}^{t}$. Then, two options could be considered for the terminal set and terminal cost. The first option is $\mathcal{X}^{t e r} \triangleq \mathcal{X}^{t}$ and $d_{\mathcal{X}^{t}}^{t e r}(x) \equiv 0$ (no terminal cost). In this case, $\mathcal{X}^{\text {ter }}$ fulfills equality (4) and $d_{\mathcal{X}^{t}}^{\text {ter }}(x)$ fulfills inequality (5) and equality (6) trivially. One drawback of this selection is that the domain of attraction of the proposed MPC is given by $\mathcal{X}_{N}\left(\mathcal{X}^{t}\right)$, which can be considerably small. A second option is $\mathcal{X}^{\text {ter }} \triangleq \mathcal{X}^{t} \oplus \delta \mathcal{X} \subseteq \mathcal{X}$ and $d_{\mathcal{X}^{t}}^{\text {ter }}(x) \geq \sum_{j=0}^{\infty} \alpha d_{\mathcal{X}^{t}}(x(j)), x(0)=x$. In this case, the real number $\delta<0$ should be selected to maximize the volume of $\mathcal{X}^{\text {ter }}$. Notice that $\mathcal{X}^{\text {ter }}$ is an ISI set (by Property 2,(4)), and so fulfill condition (4). Furthermore, a simply procedure to obtain the proposed $d_{\mathcal{X}^{t}}^{\text {ter }}(x)$, which fulfill conditions (5) and (6), was presented before.

Remark 5. It should be remarked that no prohibitive computational costs are added with the proposed MPC formulation. If the distance function is used 
as generalized distance function, the optimization problem could be re-arranged to remain a QP problem. If the modified Minkowski functional is used, an additional linear programing problem should be solved.

\section{Simulation results}

\subsection{Nominal case}

In this section some simulations results will be presented, to evaluate the proposed control strategy. To this end, a 3-state stable system of the form of $\mathcal{N}_{\text {sys }}$ is used, with matrices

$$
\begin{aligned}
A & =\left[\begin{array}{ccc}
0.5 & 0.2 & -0.3 \\
0.4 & 0.3 & 0.4 \\
-0.3 & 0.2 & 0.5
\end{array}\right], B=\left[\begin{array}{cc}
0.6 & 0.85 \\
0.5 & -0.67 \\
-0.2 & 0.4
\end{array}\right] \\
C & =\left[\begin{array}{ccc}
-0.54 & 0.8 & 0.2 \\
0.3 & -1.1 & 0.7
\end{array}\right],
\end{aligned}
$$

The constraints of the system are given by: $\mathcal{X}=\left\{x \in \mathbb{R}^{3}:\|x\|_{\infty} \leq 9\right\}$ and $\mathcal{U}=\left\{u \in \mathbb{R}^{2}:\|u\|_{\infty} \leq 1.25\right\}$. The EIS set has been selected to be $\mathcal{U}^{t}=$ $\left\{u \in \mathbb{R}^{2}:\|u\|_{\infty} \leq 0.8\right\}$, while the persistent excitation signal was selected to be a (bounded) White Noise Signal. The ISI set, $\mathcal{X}^{t}$, was selected with a volume large enough to give some robustness to the controller. Figure 2 (right) shows the relation between the feasible state space $\mathcal{X}$ (in light red) and the ISI set $\mathcal{X}^{t}$ (in dark red).

The simulations were designed to show the Re-identification operation mode of the controller. Several initial states in $\mathcal{X} \backslash \mathcal{X}^{t}$ was selected. As can be seen in Figure 2 (left), every (feasible) state is steered to the target set $\mathcal{X}^{t}$, and once the system is inside this set, the exciting procedure is activated. Furthermore, in Figure 2 (right), the state evolution inside the target set is shown. Notice that the state trajectory is around the stationary target $\mathcal{X}_{s s}^{t}$, which is also plotted (shadow plane).

Figure 3 shows the input, outputs and cost function time evolutions. Notice that there are two clear modes: first, from time $k=0$ to time $k=5$, the system is steered to the ISI set, with a decreasing cost function. Then, from time $k=6$ on, the cost function remains null, which corresponds to a persistent excitation determined by the (bounded) White Noise Signal $\mathbf{u}_{p e}$. The two time periods have been separated using different colors. Notice also, that the input is on its upper bound at the very beginning of the simulation, because the controller objective is to drive the system to the target. Furthermore, after time $k=5$, the input remain inside the EIS set $\mathcal{U}^{t}$, denoted by two dotted-lines.

\subsection{Model-mismatch case}

In this section some simulations results are presented, to evaluate the proposed control strategy in a model uncertainty scenario. To this end, a 2-state 

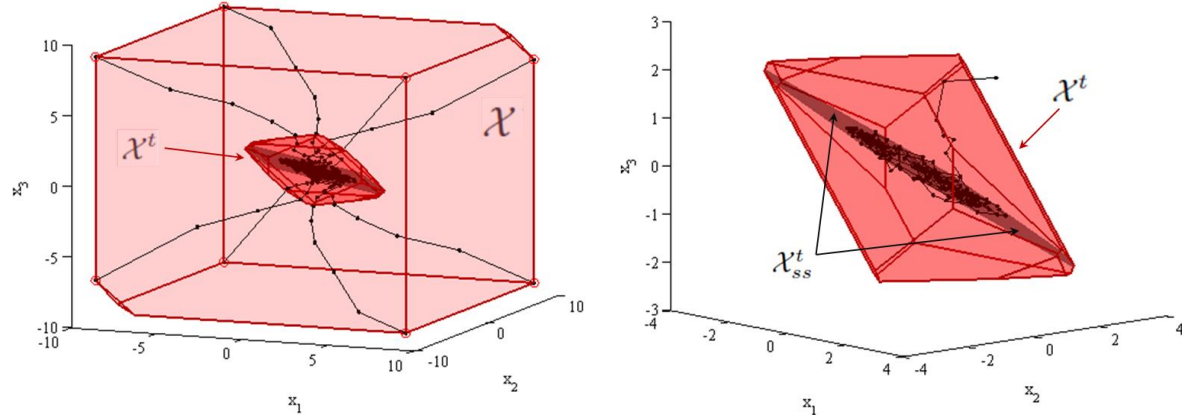

Figure 2: State evolution (left) outside and (right) inside $\mathcal{X}^{t}$.
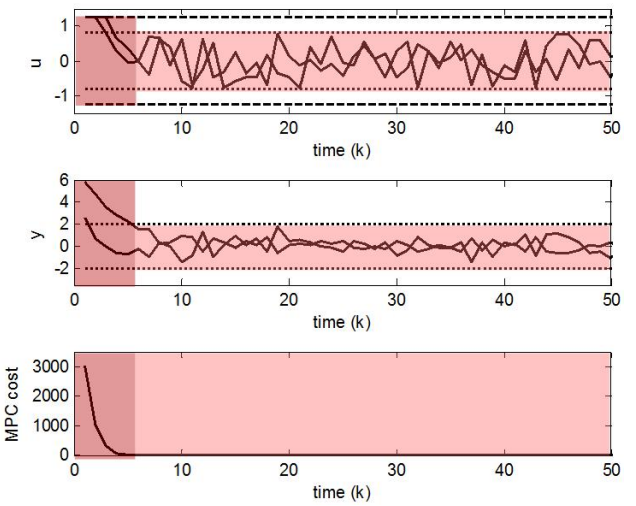

Figure 3: Input, output and cost evolution.

stable system of the form of (1) is used, with matrices:

$$
\begin{aligned}
A(w) & =\left[\begin{array}{cc}
0.42 & -0.28 \\
0.02 & 0.6
\end{array}\right]+w\left[\begin{array}{cc}
-0.6 & 0.4 \\
-0.6 & -0.85
\end{array}\right], \\
B(w) & =\left[\begin{array}{c}
0.3 \\
-0.4
\end{array}\right]+w\left[\begin{array}{l}
-0.2 \\
-0.4
\end{array}\right], \\
C(w) & =\left[\begin{array}{ll}
-0.3 & 0.6
\end{array}\right]+w\left[\begin{array}{ll}
0.1 & 0.1
\end{array}\right],
\end{aligned}
$$

and $w \in \mathcal{W}=[-0.22,0.22]$. The Nominal model is given by $A\left(w_{N}\right), B\left(w_{N}\right)$ and $C\left(w_{N}\right)$, with $w_{N}=0$, while the unknown Real model is given by $A\left(w_{R}\right)=$

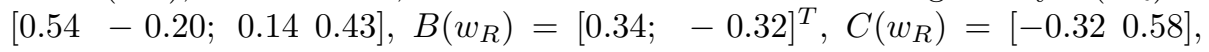
which corresponds to $w_{R}=-0.20$. The constraints of the system are given by $\mathcal{X}=\left\{x \in \mathbb{R}^{2}:\|x\|_{\infty} \leq 17\right\}$ and $\mathcal{U}=\left\{u \in \mathbb{R}:\|u\|_{\infty} \leq 1\right\}$. The EIS set has been selected to be $\mathcal{U}^{t}=\left\{u \in \mathbb{R}:\|u\|_{\infty} \leq 0.65\right\}$, while the persistent excitation signal was selected to be a (bounded) White Noise Signal.

The robust ISI set, $\mathcal{X}^{t}$, was selected according to Remark 2 (and Theorem 
1). It is given by:

$$
\mathcal{X}^{t}=\mathcal{X}^{t}(\bar{\xi}, \bar{\lambda})=\underline{\mathcal{X}}^{t}\left(w_{N}\right) \oplus \bar{\xi} \Omega_{\bar{\lambda}}=\underline{\mathcal{X}}^{t}\left(w_{N}\right) \oplus 0.85 \Omega_{0.6}
$$

where $\underline{\mathcal{X}}^{t}\left(w_{N}\right)$ is the minimal ISI set corresponding to the Nominal model, and $\Omega_{\bar{\lambda}}$ is a $\bar{\lambda}$-invariant set for $x^{+}=A\left(w_{N}\right) x$. Figure 4 shows a family of 8 minimal ISI sets, corresponding to parameters $w$ going from -0.2 to 0.1 . Furthermore, the Nominal and the Real minimal ISI sets $\underline{\mathcal{X}}^{t}\left(w_{N}\right)$ and $\underline{\mathcal{X}}^{t}\left(w_{R}\right)$, together with the ISI set $\mathcal{X}^{t}$ and $\bar{\xi} \Omega_{\bar{\lambda}}$ are plotted. The simulations were designed to show the
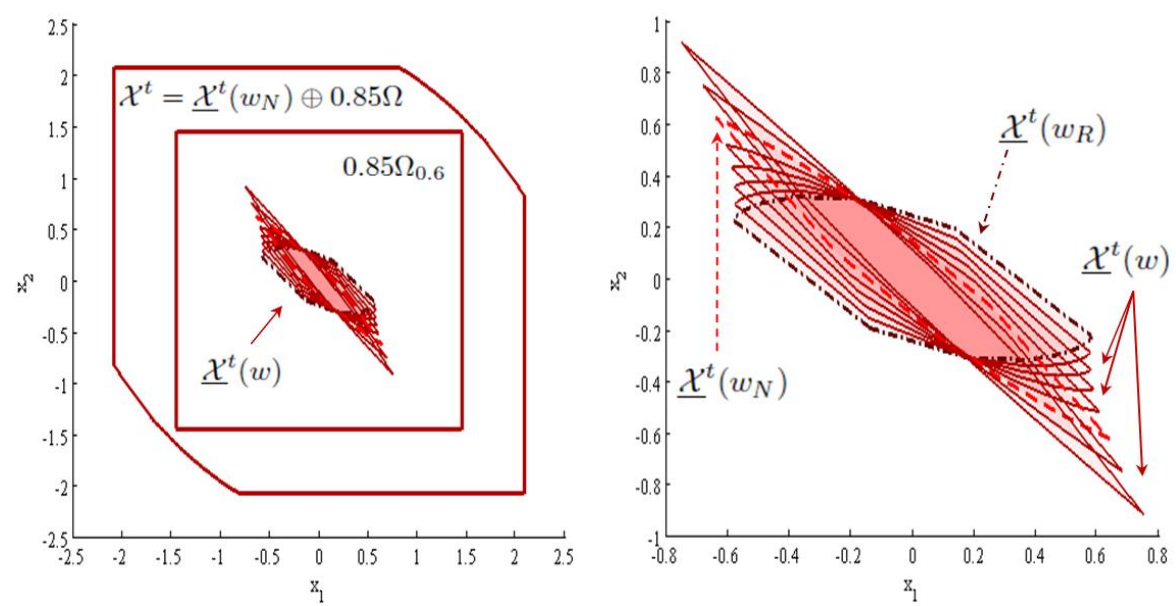

Figure 4: Family of 8 minimal ISI sets corresponding to parameters $w$ going from -0.2 to 0.1 and the Robust ISI set $\mathcal{X}^{t}$

Re-identification operation mode of the controller. To this end several initial states in $\mathcal{X} \backslash \mathcal{X}^{t}$ was selected. As can be seen in Figure 5 (left), every (feasible) state is steered to the target set $\mathcal{X}^{t}$, and once the system is inside this set, the exciting procedure is activated.

Notice that the system enters $\underline{\mathcal{X}}^{t}\left(w_{N}\right)$ and then leaves it, because this set is not associated to the Real model. In fact, the minimal ISI set for the real system is given by $\mathcal{X}^{t}\left(w_{R}\right)$ and it can be seen that once the system enters this set, it does not leaves it anymore. The important point here is that $\mathcal{X}^{t}$ is also a (non-minimal) ISI set for the Real system, and the state never leaves $\mathcal{X}^{t}$ once it enters the set. These facts are depicted in Figure 5 (right). Figures 6 (left) and 6 (right) show the input, outputs and cost function time evolutions. Figure 6 (left) has a time scale that goes only to $k=20$ to clearly show how the MPC control the system. From time $k=0$ to time $k=5$, the system is steered to the ISI set, with a decreasing cost function. Then, from time $k=5$ on, the cost function remains null, which corresponds to the injection of the persistent excitation signal $\mathbf{u}_{p e}$. The two time periods have been separated using different colors. Notice also, that the input is on its upper bound in the first time periods, because the controller tries to drive the system to the target. 

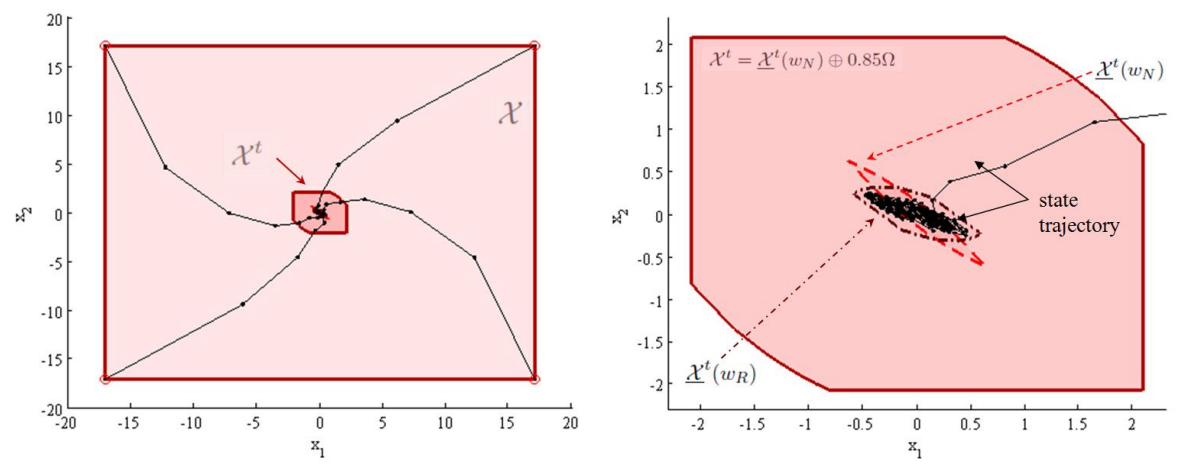

Figure 5: State evolution (left) outside and (right) inside $\mathcal{X}^{t}$.
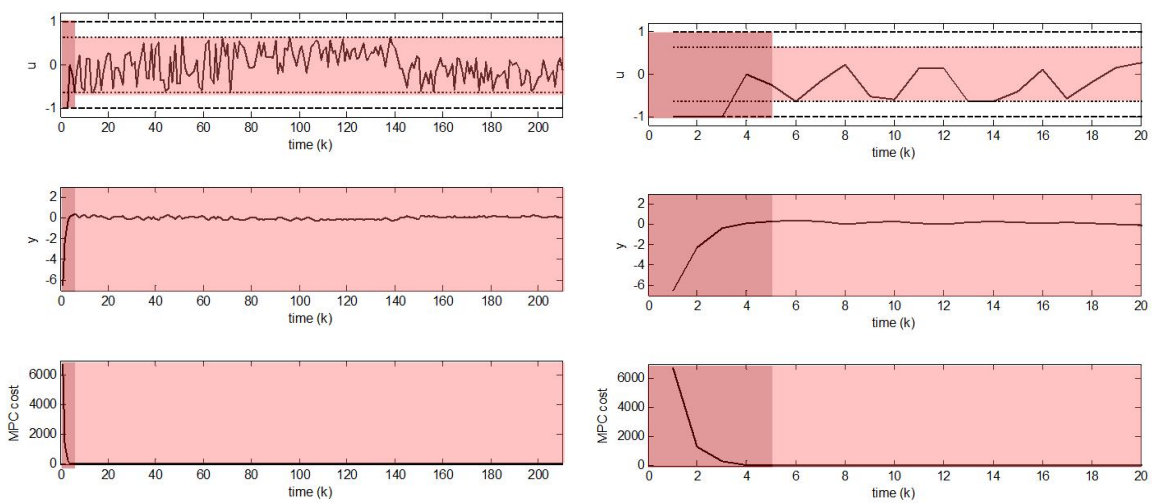

Figure 6: Input, output and cost evolution: (left) complete simulation, (right) first 20 time steps

Figure 6 (right) goes to $k=210$ to clearly show the excitation procedure. Notice that after time $k=5$, the input remains inside the EIS set $\mathcal{U}^{t}$, denoted by two dotted-lines. The excitation signal has different mean values along the simulation time, to show the invariance condition of the ISI set in this scenario.

Remark 6. The latter simulation was tested including in the loop a state observer, since in an identification scenario an observer is usually present. However, since the selected systems are of small dimension, and a well tuned observer was used, the obtained responses were not substantially different to the responses in Figure 6.

\section{Conclusions}

In this work, some results regarding a new MPC formulation suitable for closed-loop re-identification was presented. The proposed MPC guarantees persistent excitation when the system is inside a target region and guarantees re- 
cursive feasibility and closed-loop stability to this target region when the system is outside.

The key concept to pursue these two opposite objectives is the concept of attractivity of a robust invariant set, inside of which the excitation of the system can be made. In this way, the controller does not superpose the control and identification objectives, since inside the target region no control is performed and outside the target region no persistent excitation is injected to the system. To account for these properties novel generalized distance functions was proposed to construct the MPC cost function. Furthermore, to prove convergence of the method these functions have shown to be formal Lyapunov functions.

From a practical point of view, a main advantage of the method is that the identification procedure can be made as in open-loop fashion, since once the system is inside the target region, no control actions affect the system. On the other side, a preliminary drawback of the method is that a new robust invariant set needs to be computed if the target change. Future research clearly includes the study of the relation between the invariant target region and the exciting input set, in order to obtain a less conservative formulation.

\section{References}

[1] Ballin, S. L. (2008). Controlador preditivo multivariavel com restricoes de excitacao para identificacao de processos em malha fechada. Universidade de Sao Paulo. Tesis de Mestrado.

[2] Blanchini, F. and S. Miani (2008). Set-Theoretic Method in Control. Birkhauser.

[3] Ferramosca, A., D. Limon, A. H. González, D. Odloak and E. F. Camacho (2010). MPC for tracking zone regions. Journal of Process Control 20, 506516 .

[4] Genceli, H. and M. Nikolaou (1996). New approach to constrained predictive control with simultaneous model identification. AIChE Journal 42, 2857-2868.

[5] González, A. H., A. Ferramosca, G. A. Bustos, J. L. Marchetti and D. Odloak (2013). Model predictive control suitable for closed-loop reidentification. In: Proceedings of the American Control Conference (ACC).

[6] Gonzalez, A. H. and D. Odloak (2009). A stable MPC with zone control. Journal of Process Control 19, 110-122.

[7] González, A. H., E. J. Adam, M. G. Marcovecchio and D. Odloak (2011). Stable MPC for tracking with maximal domain of attraction. Journal of Process Control 21, 573-584.

[8] Green, M. and J. B. Moore (1984). Persistence of excitation in linear systems. Systems \& Control Letters 7, 351-360. 
[9] Heirung, T. A. N., B. E. Ydstie and B. Foss (2012). Towards dual MPC. In: Proceedings of the 4 th IFAC Nonlinear Model Predictive Control Conference $(N M P C)$.

[10] Kadali, R., B. Huang and A. Rossiter (2003). A data driven subspace approach to predictive controller design. Control Engineering Practice 7, 261278.

[11] Kerrigan, E. C. (2000). Robust Constraint Satisfaction: Invariant Sets and Predictive Control. PhD thesis. University of Cambridge.

[12] Kofman, E., J.A. De Dona and M.M. Seron (2012). Probabilistic set invariance and ultimate boundedness. Automatica 48, 2670-2676.

[13] Kolmanovsky, I. and E. G. Gilbert (1998). Theory and conmputation of disturbance invariant sets for discrete-time linear systems. Mathematical Problems in Engineering: Theory, Methods and Applications 4, 317-367.

[14] Larsson, C. A., L. Annergren and H. Hjalmarsson (2011). On optimal input design in system identification for model predictive control. In: Proceedings of the 50th Conference on Decision and Control $(C D C)$.

[15] Ljung, L. (1999). System identification-theory for the user. 2 ed.. PrenticeHall.

[16] Marafioti, G. (2010). Enhanced Model Predictive Control: Dual Control Approach and State Estimation Issues. PhD thesis. Norwegian University of Science and Technology.

[17] Mardi, N. A. (2010). Data-driven Subspace-based Model Predictive Control. PhD thesis. RMIT University.

[18] Overschee, P. Van and B. De Moor (1996). Subspace Identification for linear systems: theory, implementation, applications. Kluwer Academic Publishers.

[19] Rakovic, S. V. and M Lazar (2012). Minkowski terminal cost function fro mpc. Automatica 48, 2721-2725.

[20] Rawlings, J. B. and D. Q. Mayne (2009). Model Predictive Control: Theory and Design. 1st ed.. Nob-Hill Publishing.

[21] Soderstrom, T. and P. Stoica (1989). System identification. 1 ed.. PrenticeHall.

[22] Sotomayor, O., D. Odloak and L. Moro (2009). Closed-loop medel reidentification of processes under MPC whit zone control. Control Engineering Practice 17, 551-563.

[23] Wahab, N.A., R. Katebi, J. Balderud and M.F. Rahmat (2010). Datadriven adaptive model-based predictive control with application in wastewater systems. IET Control Theory and Applications 5, 803-812. 